\title{
Experimental Organism Finding
}

National Cancer Institute

\section{Source}

National Cancer Institute. Experimental Organism Finding. NCI Thesaurus. Code C137801.

An observation based on clinical, laboratory or molecular evidence, as it relates to conditions occurring in experimental organisms. 